\title{
Research on Estimation Techniques for Rician Fading Multiple- Input and Multiple-Output Channels: A Theoretical Approach
}

\author{
Wenzhun Huang and Shanwen Zhang \\ Department of Electronic Information Engineering, Xijing University, \\ $X i$ 'an 710123, China \\ huangwenzhun@xijing.edu.cn
}

\begin{abstract}
In this paper, the performance of the single-estimation and multiple-estimation is investigated in multiple-input multiple-output (MIMO) Rician flat fading channels using the traditional least squares estimator and the Bayesian minimum mean square error estimator. In high-speed mobile environment, relatively severe channel conditions change, at this point, the sender is difficult to obtain more accurate of the instantaneous channel state information, for the implementation of adaptive MIMO transmission under this scenario. The MIMO techniques can be combined with spacetime coding technique for coding diversity gain and also can use a simple multiplexing transmission in order to improve the transmission rate. The pseudo random sequence is certain, but it has many properties similar to that of the random binary data. Such as any two pseudo random sequence of cross correlation is small. In the research, we combine the basic theory of the state-of-the algorithm to propose our method. In the experimental part, we compare our method with other related state-of-the-art algorithms. The result proves the effectiveness and feasibility of the proposed method. In the near future, we plan to conduct more literature review and theoretical analysis to modify and optimize our current method.
\end{abstract}

Keywords: Channel Estimation Techniques, MIMO, Least Squares Estimator, Signal to Noise Ratios (SNRs), Bayesian Theory.

\section{Introduction}

Multiple-input multiple-output (MIMO) system provides substantial benefits in both increasing system capacity and improving its immunity to deep fading in the channel [1-3]. The fading phenomenon in the wireless communication channel was initially modelled for HF (High Frequency, 3-30MHz), UHF (Ultra HF, 300-3000 GHz), and SHF (Super HF, 3-30 GHz) bands in the 1950s and 1960s. At present, the most popular wireless channel model has been established a $2.5 \mathrm{GHz} 800 \mathrm{MHZ}$ wide channel measurement field. With the development of the modern information society, information service become the inevitable trend, gradually extended to the mobile terminal to provide traditional cellular mobile communication systems, voice business is gradually evolved to provide mobile users with Internet access, video and multimedia broadband mobile communication system of the business, this means that a new generation of mobile communication system must be able to support more than $\mathrm{MB}$ even gigabit per second transmission rate. Because has the significant advantage of a MIMO technology is considered to be new generation mobile communication system out of two big problem in the spectrum utilization and power utilization of one of the key technologies. However, even in a multiple antennas environment, for the effective and reliable to support transmission rate in excess of MB even dry megabit per second, a new generation of mobile communication system need high bandwidth, broadband 
transmission increased the frequency selective fading channel, therefore, MIMO technology must be and can effectively resist frequency selective fading block transmission technology[4-6]. However, in the out-door channel is fast fading MIMO channel, channel tracking and estimation algorithm of filter. MIMO technique uses multiple transmitter and receiver antennas to improve the performance of communication. For this reason, we also need to develop and utilize high frequency wireless resources, but due to the high frequency signal path loss and penetration loss is large, it is difficult to achieve better coverage. On the other hand, although MIMO technology has obvious advantages in wireless cellular systems, but in some specific applications, due to the restrictions on the size, cost, and the hardware of the terminal, the end user is not suitable for configure multiple antennas. In order to overcome the high frequency signal loss as well as the limitations of mobile terminal configure multiple antennas exist, can be in cellular mobile communication system, select the appropriate location set up relay nodes or select the appropriate user collaboration to improve system capacity and coverage [7-11]. With the traditional single antenna send compared to single antenna systems, MIMO wireless communication systems are faced with the more complex channel environment, the influencing factors of channel capacity more, including sending antenna, receiving antenna diameter, visual component, multipath fading and interference model, the strength of the noise, and mobile terminal speed, etc., in the process of mobile terminal, the channel between the terminal and the base station can after be determined by the factors of various types, with its capacity has a significant change, so MIMO transmission technology must be adaptive to the complex channel environment, to implement adaptive transmission use CSI is a precondition in the sending and receiving ends. In [12], the performance of the least squares (LS), scaled LS (SLS), minimum mean square error (MMSE), and relaxed MMSE (RMMSE) estimators is studied in the Rayleigh fading MIMO channel using TBCE scheme. The MMSE channel estimator has the best performance among the estimators, be-cause it employs more a-prior knowledge about the channel.

In this paper, TBCE method is studied in the flat Rician fading MIMO channels. In high-speed mobile environment, relatively severe channel conditions change, at this point, the sender is difficult to obtain more accurate of the instantaneous channel state information, for the implementation of adaptive MIMO transmission under this scenario, the sender can channel state information. Separating the main advantage of the channel model is related to its analytical expression is concise and easy to analysis, but the channel test results show that the separation of related channel models are often not able to well match the actual wireless transmission environment. In the separation of usually related and joint in the MIMO channel, the theoretically optimal transmission scheme for statistical characteristic model, namely the sending correlation matrix eigenvector determined by the direction of the parallel transmission, and according to the characteristics of the channel matrix of variance of each element in the space of power allocation, which is the theoretical basis for statistical precoding transmission technology, statistical beam forming is a special case of precoding, under low SNR, the statistics of beam forming is optimal.

In order to obtain spatial diversity and air separation multiplexing gain, also can undertake space-time coding in the statistical characteristics of the space, empty time set transmission, and air separation multiplexing transmission, namely the various open loop transmission method in statistical precoding, form the general complete adaptive transmission scheme. Optimal pilot meet with optimal, most by orthogonal channel estimation under the same conditions, and the channel estimation can be decomposed into initial least squares channel estimation and space-time post-processing cascade structure, for the statistics independent channel, space-time processing handling can be simplified as by size. Put forward a new pilot sequences design, structural features and using pilot 
sequences, significantly reduce the initial complexity channel estimation algorithm. In the following sections, we will discuss the issues in detail.

\section{The Proposed Methodology}

\subsection{The Mimo System}

To conduct the introduction to MIMO system model, we adopt the system model in [13]. $M_{T}$ and $M_{R}$ denote the transmit array and receive array, respectively. The Figure 1 illustrates the model. MIMO techniques can be combined with space-time coding technique for coding diversity gain, also can use a simple multiplexing transmission in order to improve the transmission rate, a combination of these can increase the channel capacity and transmission reliability at the same time, in addition, MIMO still can use beamforming technique to suppress the strong directional interference. In order to demodulation beamforming algorithm structure or information system needs to obtain accurate estimates of the channel state information. Due to constant characteristic of channel parameters, static channel environment of MIMO systems channel estimation is relatively simple, but in high-speed mobile application scenario, the mobile station of high-speed mobile Doppler frequency shift will cause, time varying channel to increase the complexity of MIMO channel estimation. We consider the Gaussian channel, channel so that all of the elements as Gaussian variables. If the transmitter channel is unknown, we assume that each of the antenna signal propagation $E_{s} / M_{T}$ power equality. The covariance matrix for this transmitted signal is given by the following formula:

$$
R_{s s}=\frac{E_{s}}{M_{t}} I_{M_{T}}
$$

$E_{s}$ is the power of the transmitter regardless of the number of antenna $M_{T}$ and $I_{M_{T}}$ is a unit matrix. Channel matrix $\mathrm{H}$ is $M_{R} \times M_{T}$ complex matrix. The decline of the components of the $h_{i, j}$ coefficient matrix denotes the ith which means a receiving antenna transmitting antenna. Adoption of time-varying channel estimation and the ideal channel under the condition of BER performance is far off, and the EM iterative algorithm can improve the estimation precision of time-varying channel impulse response, and thus improves the BER performance of the system. The presence of a fixed (possibly line-of-sight or LOS) component in the channel will result in Rician fading [14]. Non-blind estimation refers to the first you assess the pilot is used to obtain the position of pilot channel information for the following to prepare for the data transfer phase of the channel information blind estimation refers to not use the pilot information through the use of the information processing technology to obtain corresponding channel estimate is compared with the traditional technique of the fanaticism of estimate fanaticism estimate technology transfer efficiency of the system is greatly increased, however due to the fanaticism channel estimation algorithm generally slower convergence speed this hinders its application in the actual system as it is a half fanaticism estimate it in the data transmission efficiency and make a compromise between the convergence speed is less training sequence is used to obtain the channel information. We assume that each receiving antenna receiving power equal to the total transmission power. Since we assumed that the total received power per antenna is equal to the total transmitted power, the SNR can be written as:

$$
T=E_{S} / N_{0}
$$


As we can see, although has reduced the computational complexity, but LMMSE rule of channel estimation methods still need matrix inversion, and complicated matrix inversion algorithm, calculation is too large, seldom used in actual system. In order to further reduce the computational complexity, singular value decomposition can be used to reduce the order of the matrix. The system transmission and receive model could be expressed as:

$$
\left[\begin{array}{l}
r_{1} \\
r_{2}
\end{array}\right]=\left[\begin{array}{ll}
h_{11} & h_{12} \\
h_{21} & h_{22}
\end{array}\right]\left[\begin{array}{l}
s_{1} \\
s_{2}
\end{array}\right]+\left[\begin{array}{l}
n_{1} \\
n_{2}
\end{array}\right]
$$
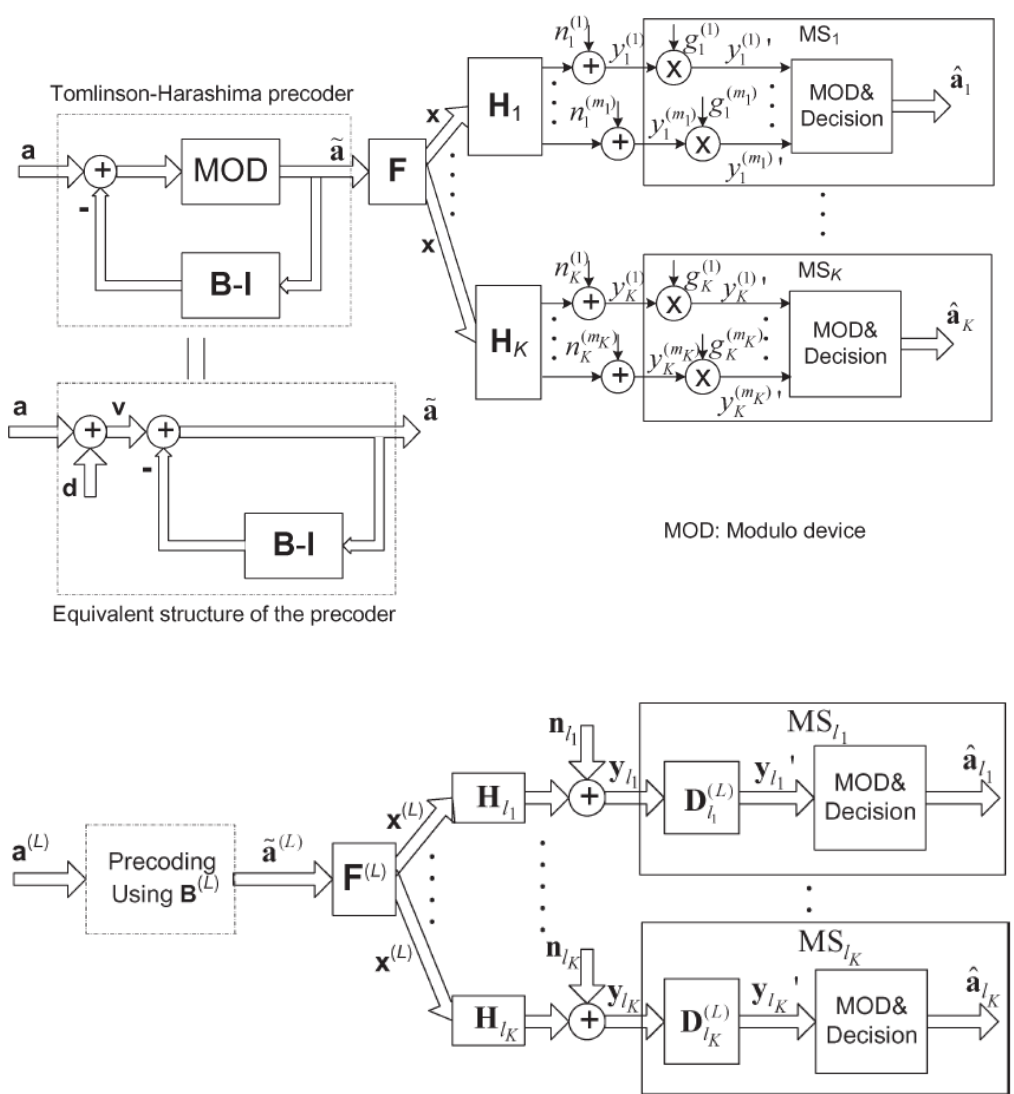

Figure 1. The MIMO System Model and the System Organization

\subsection{The System Modelling}

It is considered a MIMO system with the transmitter and $\mathrm{r}$ receiver antennas. Complete channel estimation includes the location of the channel estimation of channel estimation and data location, usually the data location in the channel estimation is the pilot location according to estimate the channel state information, by interpolation method. As a result of the known data symbols insert, a certain bandwidth resources, and if the channel changes quickly, in order to obtain a better channel estimation, you need to use a lot of pilot information, will seriously affect the transfer efficiency of the system. Blind/half fanaticism estimates, compared with other two algorithms, improve the system transmission efficiency; But since there is no reference information, estimation algorithm mostly need to deal with the complex method, the algorithm computational complexity, slow convergence speed, and is suitable for the slowly varying channel, only very limited in practical application. Channel estimation algorithm based on decision feedback is generally applicable to the slowly varying channel, it is to use a small amount of known information to estimate the current state 
of the channel, as a symbol of the channel information to achieve balanced, then after demodulation data symbols as known information to estimate, as a symbol of the channel of information, in turn, recursive, estimates the whole channel. Then, the results are used for data detection which could be expressed as the formula 4:

$$
Y=H X+V \quad R_{V}=E\left\{V^{H} V\right\}=r I_{n_{p}}
$$

Where $\mathrm{X}$ and $\mathrm{V}$ are the complex $\mathrm{t}$-vector of transmitted sequences on the $\mathrm{t}$ transmit antennas and $\mathrm{r}$-vector of additive receiver noise, respectively. The elements of noise matrix are independently and identically distributed complex Gaussian random variables with zero-mean and unit variance. After transformation and string the modulation to the emission spectrum, and then launch into the wireless channel. In this paper, we mainly consider the influence of multipath channel. Usually, the multipath channel can be modeled as FIR filter and assume that the signal reaches the receiver of the multipath channel. The matrix $H_{R a y}$ explains the Rayleigh component of the channel and the matrix $H_{\text {Los }}$ describes the channel mean value or LOS component of the channel. It is defined in the formula 5.

$$
M=E\{H\}=\sqrt{K /(K+1)} H_{1}+\sqrt{1 /(K+1)} H_{2}
$$

Therefore, the correlation matrix of the channel can be calculated as follows:

$$
\begin{aligned}
& R_{H}=E\left\{H^{H} H\right\}=\frac{1}{1+K} R_{H_{R a y}}+\frac{K}{1+K} H_{L O S}^{H} H_{L O S} \leq H_{L O S}^{H} H_{L O S} \\
& R=E\left\{H H^{H}\right\}=\frac{K}{1+K} R_{H_{R a y}}+\frac{1}{1+K} H_{L O S}^{H} H_{L O S} \leq R_{H_{R a y}}
\end{aligned}
$$

\subsection{The Single Channel Estimation}

In this section, it is assumed that the ship is used for channel estimation, the number of $\mathrm{N}=1$. Initially, the LS channel estimator is studied. In wireless mobile communication, the channel state information is unknown and time-varying, which requires the real-time accurate to estimate the amplitude and phase of transmission channel, in order to accurately to send the data demodulation. In the use of high-density constellation points of OFDM system, the channel state estimation accuracy significantly affects the performance of the system. Then, the performance of the Bayesian MMSE channel estimators is examined. Due to the larger peak to average power ratio, causes the RF amplifier power efficiency is low. Due to OFDM system in the first place to need to send data in the inverse Fourier transform processing, when the linear treatment increased the in-phase component, after stacking synthetic signal can produce larger peak power, also will bring the larger peak average power ratio. We firstly analyze the LS channel estimator, for linear model of formula 1 and the LS channel estimator is formulated as:

$$
\hat{H}_{L S}=Y X^{H}\left(X X^{H}\right)^{-1} \quad \text { S.T.Min }\left\{(Y-H X)^{H}(Y-H X)\right\}
$$

To find out the optimum solution for the problem, through reviewing the following literatures we find out the core technique [15-26]:

$$
h_{L S}=\left(A^{H} A\right)^{-1} A^{H} y
$$


In the formula, to ensure the selectivity and frequency selective channel of time can have very good estimate effect, then inserted pilot frequency interval as small as possible, but if you insert number too much, the band utilization rate of the system reduced, and increase system overhead. Sparse reconstruction using mainstream algorithm, it is in reconstruction has a reasonable compromise between performance and complexity, the most widely in the practical application. Pilot inserts number, therefore, should not only consider the accuracy of channel estimation, also want to consider the bandwidth utilization of system. The Bayesian MMSE channel estimator of $\mathrm{H}$ is given by:

$$
\hat{H}_{M M S E}=M+(Y-M X) A+\max \left|A_{m}^{H} A_{n}\right|
$$

The performance of the MMSE channel estimator is measured by the error matrix $\varepsilon=H-\hat{H}_{M M S E}$ whose probability density function (pdf) is Gaussian with zero mean and the following covariance matrix and then, the Then through and string conversion and constellation points extraction solution map data. Is not hard to find, the effect of channel equalization is closely related to the accuracy of the channel estimation, which directly depends on the density of pilot and interpolation method MMSE estimate error is modeled as the following formulas:

$$
\begin{gathered}
C_{\varepsilon}=R_{\varepsilon}=E\left\{\varepsilon^{H} \varepsilon\right\}=\left(C_{H}^{-1}+\frac{1}{r} X X^{H}\right)^{-1} \\
J_{M M S E}=E\left\{\operatorname{tr}\left(\varepsilon^{H} \varepsilon\right)\right\}=E \cdot \max _{t \in \Omega}\left|\sum_{l=1}^{K} \exp (-j 2 \pi N)\right|
\end{gathered}
$$

To minimizing 11 which is subject to the transmitted power constraint, After considering the pilot inserted into the quantity, then consider pilot inserted into the form, even inserted with non-uniform insert have any influence on the performance of channel estimation, etc. we adopt the Lagrange multiplier method. The minimized MSE is denoted as:

$$
J_{M M S E}^{\min }=E \cdot \max _{t \in \Omega}\left|\sum_{l=1}^{K} \exp (-j 2 \pi N)\right|-E \cdot \min _{t \in \Omega}\left|\sum_{l=1}^{K} \exp (-j 2 \pi N)\right|
$$

When meet restricted isometric characteristics measurement matrix, for all signals with sparse characteristics can refactor with matching pursuit algorithm, but the use of the orthogonal matching pursuit algorithm is relatively limited, it is only applicable to fixed signal. But short time needed for fast orthogonal matching pursuit algorithm therefore become one of the commonly used reconstruction algorithm.

\section{Multiple Channel Estimation}

\subsection{Prior Knowledge}

In order to improve the performance of the estima-tors, in mobile communication systems, based on training sequence channel estimation is made by sending training sequences, the training sequence through wireless communication channel, the receiver based on training sequence, and the received signal to estimate the channel impulse response values. The pseudo random sequence is certain, but it has many properties similar to that of the random binary data. Such as any two pseudo random sequence of cross correlation is small, the original sequence and translation after serial correlation. 
Mobile communication system is usually not ideal in the channel, in the process of transmission is fading, and the change of the channel uncertainty, obtain accurate channel state information is the premise of giving full play to the advantages of wireless communication, need to channel estimation and channel state information acquired technology to complete. Most modern mobile communication system to accurately complete by pilot symbol aided channel estimation and tracking in time the change of the wireless channel.

Suppose that $\mathrm{N}$ estimates of the MIMO channels are obtained based on the training matrices $X_{1}, \ldots, X_{N}$. So for a given environment and system, to get a higher channel estimation precision will need to design the reasonable training sequence. Channel estimation is refers to the receiver according to the received signal transmission characteristics of channel estimation, and make the estimate result and the actual channel transmission characteristics as close as possible. The results are combined in the following linear method with the MSE:

$$
\hat{H}_{M E}=\sum_{n=1}^{N} a_{n} \hat{H}_{n} \quad P_{o p t}=\arg \min _{p} f(P)
$$

We could then transfer the optimization problem to the following:

$$
\min _{a_{1}, \ldots, a_{N}}=E\left\{\left\|H-\sum_{n=1}^{N} a_{n} \hat{H}_{n}\right\|_{F}^{2}\right\} \quad S . T \cdot \sum_{n=1}^{N} a_{n}=1
$$

In the common channel estimation based on training sequence, the training sequences are modulator before training sequences, and the sequence of the design after training sequence is modulator, they are all real number sequence. With the traditional channel estimation that is commonly used in the process of $\mathrm{m}$ sequence estimation performance comparison, as a result of $\mathrm{m}$ sequence is usually a binary, in order to maintain the variable consistency, first the $\mathrm{m}$ sequence is needed here after different modulation system is transformed into real number sequence. Will be designed by three kinds of real number sequence by BPSK demodulation respectively, due to the sequence in the process of demodulation are discrete, the carrier drop sampling by sequence with multiplier multiplication, ruling by low-pass filter and then get the binary sequence after demodulation.

\subsection{Multiple Ls Estimation}

The traditional LS algorithm without using the characteristics of sparse channel itself, estimated price is bigger. Compressed sensing theory shows that when the signal is sparse or compressible, use frequency is far lower than the Nyquist rate of sampling signal, through a small amount of signal projection value, can realize accurate signal or similar to refactoring. M sequence because of its good autocorrelation characteristic as the training sequences for channel estimation, but not from the channel estimation and optimization guidelines to improve the channel estimation accuracy. Based on the theory of compressed sensing in order to get better effect of channel estimation for the purpose of design is a new training sequence, and by the simulation results can be concluded that the design of the above three kinds of sequence can achieve good effect of channel estimation, and a sequence of Bernoulli distribution on the best effect. With the LS estimator, we could rewrite it as:

$$
\hat{H}_{L S}=H+V X^{H}\left(X X^{H}\right)^{-1}
$$

Using (15), the error of the multiple LS estimation will be written as: 


$$
J_{\text {MultipleLS }}=E\left\{\left\|H-\sum_{n=1}^{N} a_{n}\left(H+V_{n} X_{n}^{H}\left(X_{n} X_{n}^{H}\right)^{-1}\right)\right\|_{F}^{2}\right\}
$$

Comparing (16) and (11), it is seen that in the multiple LS estimation, the error reduces by the number of sub-blocks $\mathrm{N}$ which is used for channel estimation. It is notable that the error (16) is independent of $p_{n}$, the transmitted power during the $\mathrm{n}$-th training period. Generally speaking, channel is refers to the signal after the process of transmission through the media. In the process of signal transmission, the transmission of signal will be affected by the channel, make its phase and frequency and amplitude have some change. The so-called channel estimation is at the receiving end according to the received signal to estimate the channel impulse response value. It means that for uniform training powers and non-uniform training powers during $\mathrm{N}$ training periods, the error is the same. Furthermore the MSE of multiple Bayesian MMSE channel estimator is expressed as:

$$
\begin{aligned}
& J_{\text {MultipleLS }}=E\left\{|| \sum_{n=1}^{N} a_{n} V_{n} X_{n}^{H}\left(X_{n} X_{n}^{H}\right)^{1} \|_{F}^{2}\right\}=r \operatorname{tr}\left\{\sum_{n=1}^{N}\left|a_{n}\right|^{2} E_{n}\right\} \\
& J_{L S}=r \operatorname{rt}\left\{\sum_{n=1}^{M}\left|a_{n}\right|^{2} E_{n}\right\}+\frac{1}{2} \operatorname{rtr}\left\{\sum_{n=1}^{N}\left|a_{n}\right|^{2} E_{n}\right\}
\end{aligned}
$$

Comparing (17) and (8), found in a number of LS estimation, error reduction in the number of ships used for channel estimation of N. It is important to note that error (17) were independent of each other. When using pilot channel estimation, we usually need to insert the pilot in OFDM symbol and the receiver can be used to the received signal to the pilot's position for channel estimation, then estimates the whole channel frequency response. Thus make the calculation process more simple and effective to reduce the complexity of operation, at the same time, by the result of simulation shows that this method does not bring great influence on the performance of the algorithm. It means that for uniform training powers and non-uniform training powers during $\mathrm{N}$ training periods, the error is the same.

\subsection{Multiple Bayesian MMSE Estimation}

Compared with the LS algorithm, LMMSE algorithm has the strong antiinterference characteristics, so the estimation performance is greatly improved, but meanwhile LMMSE algorithm also has shortcomings, in the process of computing the need for matrix calculations, so the computational complexity of this algorithm was greatly increased. After deduction, to shift to the mathematical model of compressed sensing, and through the theoretical analysis it is concluded that the compressed sensing is feasible in the channel estimation. Put forward a kind of sparse channel estimation algorithm based on the system, the algorithm adopts the hyperbolic tangent function to approximate Si norm, in view of the algorithm convergence speed and the iteration step length selection in the shortfalls, quasi-newton method is used to solve the optimal value, and through the line search technique to determine the iteration step length, the proposed algorithm can under the condition of the sparse degree of unknown channel impulse response channel estimation to get good results. We could rewrite the MMSE channel estimator and the MSE and multi-Bayesian MMSE channel estimator as the following:

$$
\hat{H}_{\text {MMSE }}=M+(H-M) X A+V A
$$




$$
J_{\text {MultipleMMSE }}=E\left\{\left\|H-\sum_{n=1}^{N} a_{n}\left(M+(H-M) X_{n} A_{n}+V_{n} A_{n}\right)\right\|_{F}^{2}\right\}
$$

OMP algorithm is the same as the MP algorithm of matching atom selection criterion, the difference is that each iteration of the selected atoms are in the process of processing, thus ensuring the iteration of optimality, effectively reduced the number of iterations, improves the convergence speed. OMP algorithm computational complexity is still high, can one-time multiple selected atoms in each iteration, rather than a single atom, so it can reduce the iteration times and improve the speed, but at the cost of this at the expense of reconstruction precision shown as the follows.

$$
\min \left\|\left\langle f, g_{i}\right\rangle\right\|_{0} \quad \text { s.t. } \quad f=\sum_{t=0}^{K-1}\left\|\left\langle f, g_{i}\right\rangle\right\|
$$

Considering the MMSE channel estimation usually need a channel of the second order statistics, such as channel covariance matrix, etc., and based on the sparse channel estimation does not need the second order statistics, as a result, the algorithm in this paper and also don't need the second order statistics of the LS channel estimation is compared.

\section{Experiment and Simulation Result}

\subsection{Set-Up of the Experiment}

In this section, the performance of the LS and MMSE estimators is numerically examined in the case of SE and ME. First, the single-estimation (SE) is considered and the minimum MSE is obtained for LS and MMSE estimators under optimal training. Digital information from simulation of the source is the way of sampling, this is the digital signal processing of mould and real-life MoNiHua. Nyquist sampling theorem is pointed out that in order to accurately reconstruct the original signal, sampling frequency must be greater than or equal to twice the signal bandwidth. Then, multipleestimation (ME) is investigated in these estimators. In ME case, the multiple estimates of the channel during received $\mathrm{N}$ sub-blocks are combined optimally. The optimal weight coefficients are achieved for both estimators. Furthermore, the minimum MSE under optimal training is obtained for aforementioned estimators. The emergence of the theory of compressed sensing makes it possible to solve the problem. Compressed sensing theory suggests that if the signal in a transform domain is sparse, and the signal can be compressed, so the available will be an observation matrix transformation project of high-dimensional signals to a lower dimensional space, this observation is not related to matrix and transformation matrix. By solving optimization problem is then able to high probability from these a few projection to recover the original signal, by demonstrating that the small amount of projection contains enough information to reconstruct the signal. Therefore under the framework of compressed sensing theory, the sampling frequency is determined by the content and structure of information in the signal instead of the bandwidth of the signal. As a performance measure, it is considered that the channel MSE is normalized by the average channel energy as:

$$
N M S E=\frac{E\left\{\|H-\hat{H}\|_{F}^{2}\right\}}{E\left\{\|H\|_{F}^{2}\right\}}
$$

Sparse channel impulse response coefficient is zero, only a handful of tap in most of the remaining tap coefficient is zero or nearly to zero. Same as [2], the elements of the covariance matrix of the channel are defined as follows: 


$$
\left[c_{H}\right]_{k, 1}=\frac{r}{1+K} \rho^{|k-l|}, 0 \leq|\rho| \leq 1
$$

\subsection{Experiment Simulation}

The Figure 2 shows Normalized MSE (NMSE) of the LS channel estimator with optimal training versus SNR in the case of SE and ME. According to this figure, increasing the number of the sub-blocks $\mathrm{N}$ results in a lower error of the estimation. In the experiment, OFDM is 128, the number of neutrons carrier data for QPSK modulation. It is assumed that the channel within a frame OFDM symbol was a constant, channel length of 100 , the sparse degree of 10 . Take guide frequency $\mathrm{P}=60$ simulation, compare various algorithms of mean square error (MSE), the mean square error (MSE) of each algorithm 100 times to experiment on average. MSE optimal pilot channel estimation for statistical precoding pilot, the pilot should send relevant matrix eigenvector determined the direction of the parallel transmission, on the basis of channel correlation matrix eigenvalue and power allocation. Subprime pilot power allocation scheme is put forward in approaching at the same time, the performance of the optimal power allocation significantly reduce the implementation complexity. In the Figures 3, Figure 4 and Figure 5, we illustrate the experimental result.

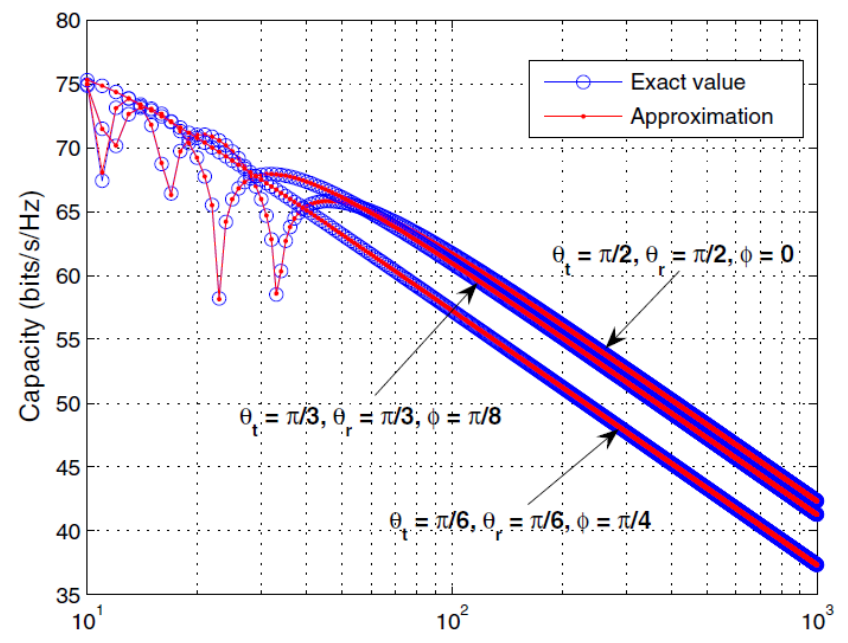

Figure 2. The Channel Estimation Simulation for the Proposed
Methodology

\section{Conclusion and Summary}

In this research paper, we conduct research on the estimation techniques for Rician fading multiple-input and multiple-output channels. With the development of the modern information society, information service becomes the inevitable trend, gradually extended to the mobile terminal to provide traditional cellular mobile communication systems. Non-blind estimation refers to the first you assess the pilot is used to obtain the position of pilot channel information for the following to prepare for the data transfer phase of the channel information blind estimation refers to not use the pilot information through the use of the information processing technology to obtain corresponding channel estimate is compared with the traditional technique of the fanaticism of estimate fanaticism estimate technology. In the experimental part, we compare our method with other related state-of-the-art algorithms. The result proves the effectiveness and feasibility of the proposed method. In the future, we have scheduled to conduct more related research. 

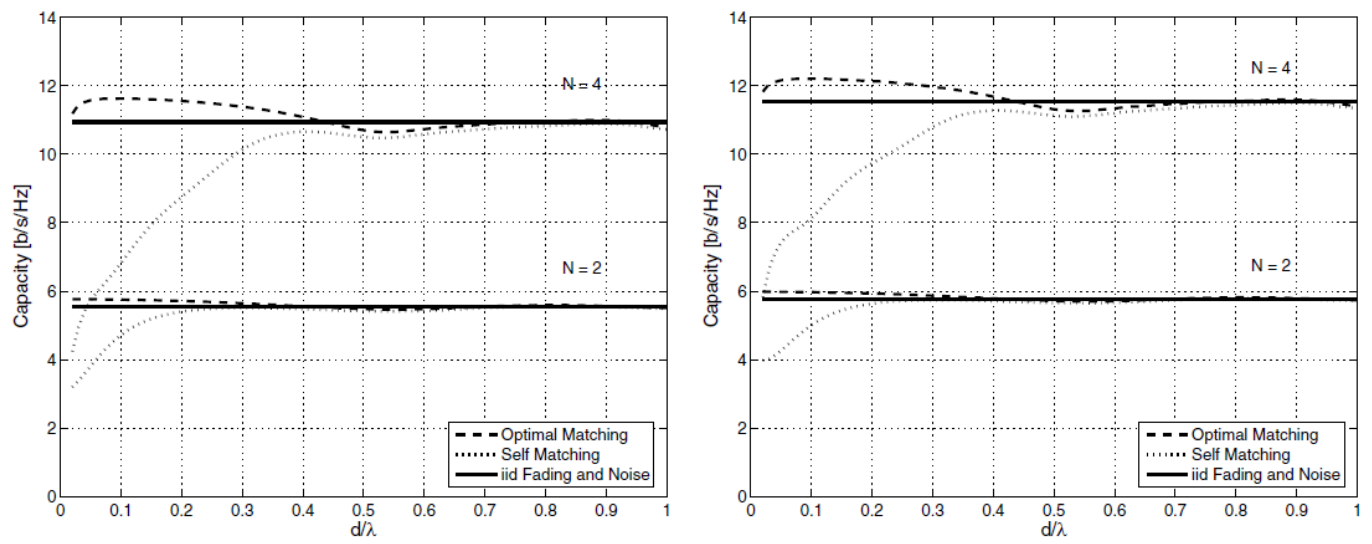

Figure 3. The Experimental Simulation Set One
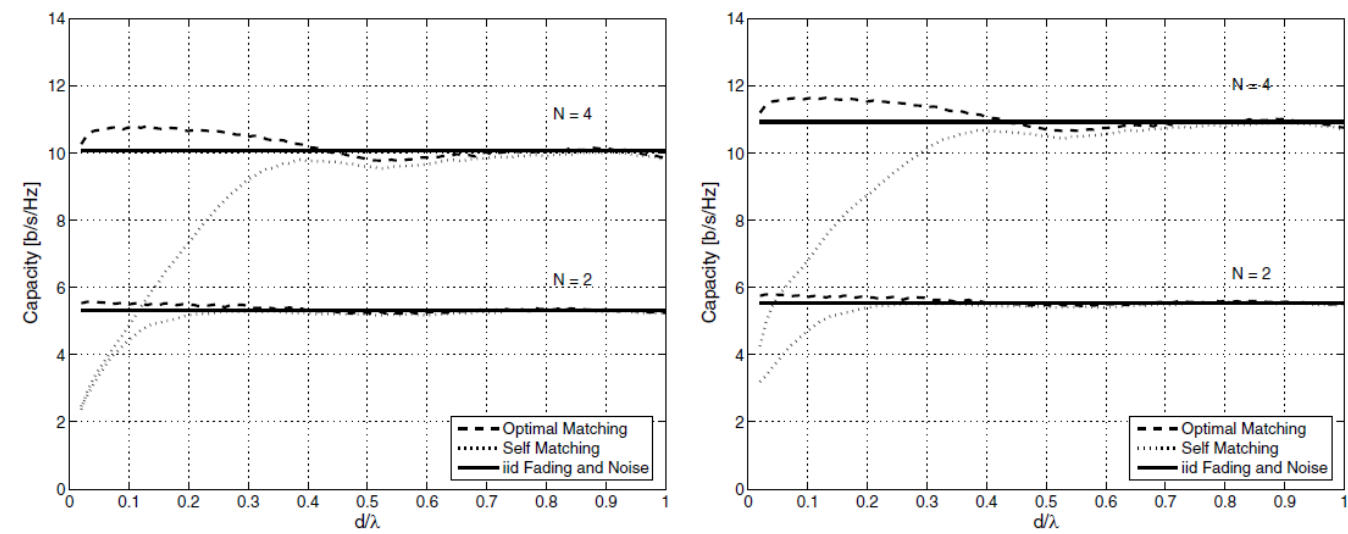

Figure 4. The Experimental Simulation Set Two
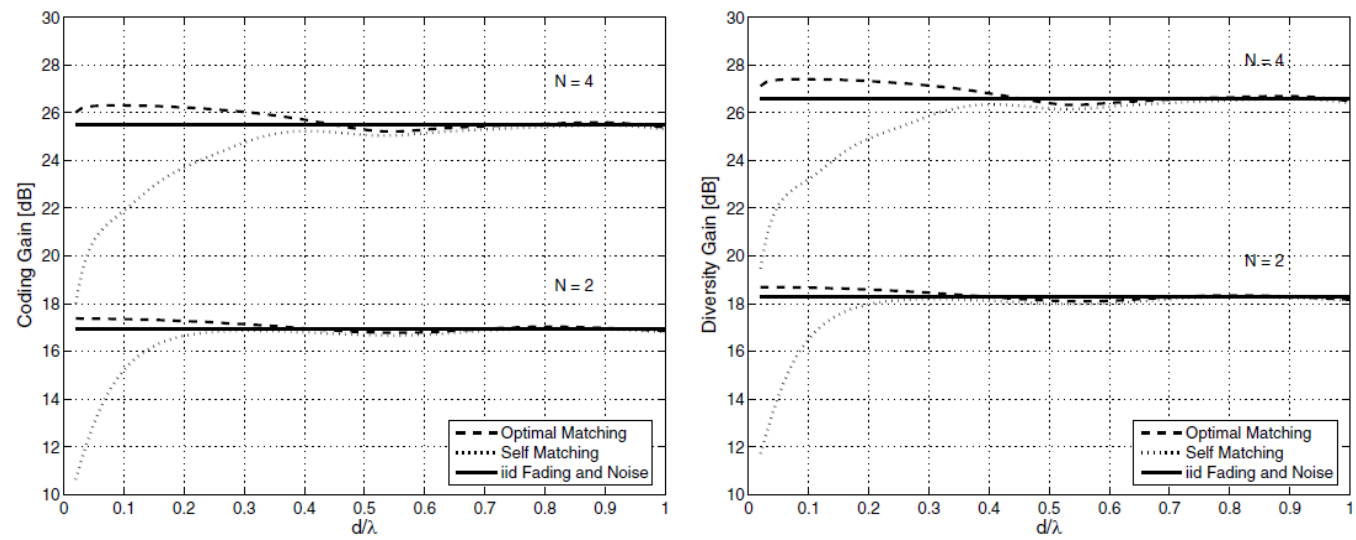

Figure 5. The Experimental Simulation Set Three

\section{Acknowledgement}

This work is financially supported by the scientific research program of Shaanxi Provincial Education Department (Program No. 14JK2156). 


\section{References}

[1] J. Du, C. Yuan and J. Zhang, "Low complexity PARAFAC-based channel estimation for nonregenerative MIMO relay systems", IET Communications, vol. 8, no. 12, (2014), pp. 2193-2199.

[2] M.A. Hanif, M.H. Lee and S.S. Song, "A noble circularly symmetric Gaussian covariance matrix based channel estimation scheme for large-scale MIMO systems", 2014 International Conference on Information Networking (ICOIN), (2014), pp. 116-119, Phuket(TH).

[3] W. Shen, "Spatially correlated channel estimation based on block iterative support detection for large-scale MIMO", arXiv preprint arXiv:1410.5331, (2014).

[4] W. Shen, "Spatially correlated channel estimation based on block iterative support detection for large-scale MIMO", arXiv preprint arXiv:1410.5331, (2014).

[5] G. Yang, "Throughput Optimization for Massive MIMO Systems Powered by Wireless Energy Transfer", arXiv preprint arXiv:1403.3991, (2014).

[6] R.O. Adeogun, D.T. Paul and A.D. Pawel, "Parametric channel prediction for narrowband MIMO systems using polarized antenna arrays", Proceeding on IEEE Vehicular Technology Conference Spring, (2014).

[7] M. Amrita, G.R. Jagannatham and K.J. Aditya, "Random Parameter EM-Based Kalman Filter (REKF) for Joint Symbol Detection and Channel Estimation in Fast Fading STTC MIMO Systems", (2014), pp. 1-1.

[8] J. Seo, "Pilot beam sequence design for channel estimation in millimeter-wave MIMO systems: A POMDP framework", arXiv preprint arXiv:1409.8434, (2014).

[9] G.R. Patil and V.K. Kokate, "Joint channel estimation and data detection for SFBC MIMO OFDM wireless communication system", 2014 International Conference on Advances in Computing, Communications and Informatics (ICACCI), IEEE, (2014), pp. 2565-2570; Delhi(IN).

[10] A. Hu, "Pilot design for large-scale MIMO system with semi-blind channel estimation", IEEE Transactions on Communications, (2014).

[11] X. Li, S. Zhou, Y. Yan, Z. Xiao and J. Wang, "Cluster Information based User Scheduling for Multiuser MIMO Systems", IEEE Military Communications Conference (MILCOM), (2014), pp. 1580-1585; Baltimore, MD(US).

[12] Reshamwala, S. Nirmalkumar, P. S. Suratia, and K. S. Satish, "Time-Delay Neural Network for Smart MIMO Channel Estimation in Downlink 4G-LTE-Advance System", (2014).

[13] L. Zhou, T. Ratnarajah and X. Jiang, "Channel estimation and performance analysis of beamspace MIMO systems", European Conference on Networks and Communications (EuCNC), (2014), pp. 1-5, Bologna(IT).

[14] J.J. Tan, "Simulation of MIMO channel characteristics for indoor visible light communication with LEDs", Optik-International Journal for Light and Electron Optics, vol. 125, no. 1 (2014), pp. 44-49.

[15] M.P. Dhake, "Adjoint based compressible Euler shape optimizaion code for propeller driven aircraft", Diss. JNCASR, (2014).

[16] C.U.I. Meng, "Maximizing Drilling Performance With Real-Time Surveillance System Based on Parameters Optimization Algorithm", Advances in Petroleum Exploration and Development, vol. 8, no. 1, (2014), pp. 15-24.

[17] B. Xu, "On reverse Hilbert-type inequalities", Journal of Inequalities and Applications, vol. 1, (2014), p. 198.

[18] F. John, "Extremum problems with inequalities as subsidiary conditions", Traces and Emergence of Nonlinear Programming, Springer Basel, (2014), pp. 197-215.

[19] M.J. González, T. Jurado and M. Naldini eds, "Gender inequalities in southern Europe: woman, work and welfare in the 1990s", Routledge, (2014).

[20] W. Beckner, "Geometric inequalities in Fourier analysis", Essays on Fourier analysis in honor of Elias M. Stein, (2014), pp. 36-68.

[21] R. Shaw, M. Benzeval and F. Popham, "Do financial strain and labour force status explain why Nordic countries have wide health inequalities relative to other European countries", The European Journal of Public Health, vol. 24, no. 2, (2014), pp. 164-014.

[22] M.P. Johan, "Trends in inequalities in premature mortality: a study of 3.2 million deaths in 13 European countries", Journal of epidemiology and community health, (2014).

[23] A.N. Aziz, "Structured Mathematical Modeling of Industrial Boiler", Journal of Engineering and Technological Sciences, vol. 46, no. 1, (2014), pp. 102-122.

[24] K. Leder, "Mathematical modeling of PDGF-driven glioblastoma reveals optimized radiation dosing schedules", vol. 156, no. 3, (2014), pp. 603-616.

[25] D.S. Chastukhin, A.V. Borodin and B.I. Khodorov, "Mathematical modeling of delayed calcium deregulation in brain neurons caused by hyperstimulation of glutamate receptors", Biophysics, vol. 59, no. 2, (2014), pp. 236-247.

[26] V.E. Katzourakis and V.C. Constantinos, "Mathematical modeling of colloid and virus cotransport in porous media: Application to experimental data", Advances in Water Resources, vol. 68, (2014), pp. 62-73. 

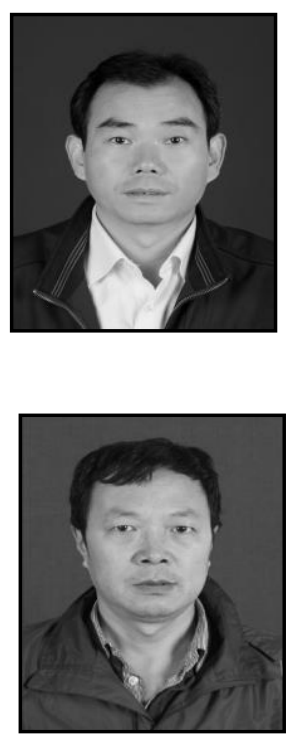

\section{Authors}

Wenzhun Huang, He received his B.S. and M.S. degrees in Communication and information system from Air Force Engineering University, China, in 1997. He received his Ph.D. degree in Information and communication engineering from Northwest Polytechnic University, China, in 2010. He is an associate professor in Xijing University. His research interests include information processing, anti-jamming technology, wireless communication system and IoT technology.

Shanwen Zhang, He received his B.S. degree in mathematics from Northwest University, China, in 1988. He received M.S. degree in applied mathematics from Northwest Polytechnic University, China, in 1995 . He received Ph.D. degree in electromagnetic field and microwave from Air Force Engineering University, China, in 2001. He is a professor in Xijing University. His research interests include wavelet transforms, rough sets, genetic algorithm, pattern recognition and manifold learning. 
International Journal of Future Generation Communication and Networking Vol. 9, No. 3 (2016) 